\title{
One-Dimensional Model for the Ultrasonic Response of Resin-Filled Gaps in Automated Tape Layup Composites
}

\author{
Patrick H. Johnston \\ Nondestructive Evaluation Sciences Branch \\ NASA Langley Research Center \\ Hampton, Virginia, USA 23681-2199 \\ patrick.h.johnston@nasa.gov
}

\author{
Peter D. Juarez \\ Nondestructive Evaluation Sciences Branch \\ NASA Langley Research Center \\ Hampton, Virginia, USA 23681-2199 \\ peter.d.juarez@nasa.gov
}

\begin{abstract}
Automated tow placement has become a widelyused fabrication technique, especially for large aerospace structures. Robotic heads lay down strips (tows) of preimpregnated fiber along programmed paths. The intention is to lay adjacent tows abutted to one another, but sometimes a gap is left between a tow and the previously-placed tow. If a tow gap exists, it fills with resin during cure, forming a fiber-free volume. In immersion ultrasonic pulse-echo measurements of a cured laminate, the gap can be observed to produce a noticeable echo, without significantly attenuating the back-wall reflection of the laminate. To understand this behavior, we considered a onedimensional model of the composite laminate, with a thin layer having the ultrasonic sound speed and density of neat resin, sandwiched between two layers of material having the sound speed and density of fiber-reinforced composite and surrounded on both sides by water. Neglecting attenuation, we considered the transmission and reflection coefficients of each interface, as well as that of the thin resin layer. Using the initial water/composite reflection as a reference, we computed the relative magnitude of the back surface/water reflection in the presence and in the absence of a resin-only layer, as well as the relative magnitude of the reflection arising from a thin resin layer in composite. While the one-dimensional model did not fully match the measurements, it did qualitatively explain the observed behavior.
\end{abstract}

Keywords—composites, automated fiber tow placement

\section{INTRODUCTION}

Automated tow placement is a widely-used fabrication technique for large aerospace structures. Robotic tape placement machines, such as shown in Fig. 1, lay down strips of pre-impregnated fiber tows along programmed paths. Adjacent tows should ideally be abutted to one another, but, occasionally, a gap occurs between tows. The presence of tow gaps has been shown to weaken a structure; e.g., a simulation study showed up to $32 \%$ strength reduction [1] and an experimental study found $7 \%-12 \%$ strength reduction [2].

The purpose of this work was to investigate a phenomenon observed during ultrasonic pulse-echo scanning of tow-placed laminates. It was noted during scanning of a

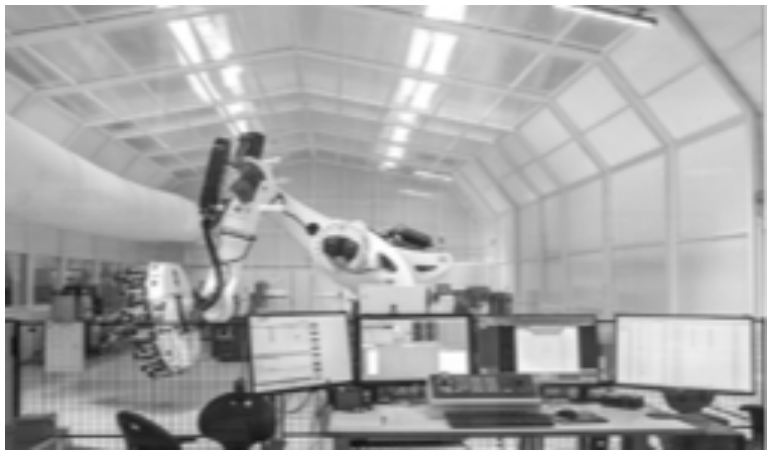

Fig. 1. Photograph of an automated tape placement robot.

panel having intentional tow gaps, fabricated to support an investigation of in situ thermographic detection of tow gaps [3], that the gaps produced an ultrasonic echo, while the underlying back surface reflection was minimally changed or unchanged. This seemed unusual, as discontinuities producing echoes usually also attenuate the sound.

This phenomenon was also observed in a panel, which had previously been inspected in a different lab using a back-wall $\mathrm{C}$-scan and ruled clear of anomalies, during a follow-up scan in our lab. The possibility of missing potentially important tow gaps motivated an effort to better understand the phenomenon. Fig. 2 presents a C-scan of this panel, time-gated interior to the laminate to show the echoes from several tow gaps..

\section{APPROACH}

In order to better understand the physics underlying this measurement, we sought a simple physical model to describe the measurement. To begin, we needed a physical description of the material state under interrogation. Fig. 3 presents a schematic cross-section of the tow gap geometry. When nominally placed, adjacent tows cure into continuous lamina as depicted in Fig. 3A. Fig. 3B depicts a tow gap, of width only a few laminae thicknesses $d$. In this case, the overlying courses of tape may bridge the gap, without distortion, allowing resin to flow into the gap and cure to form a one-laminae-thick layer 


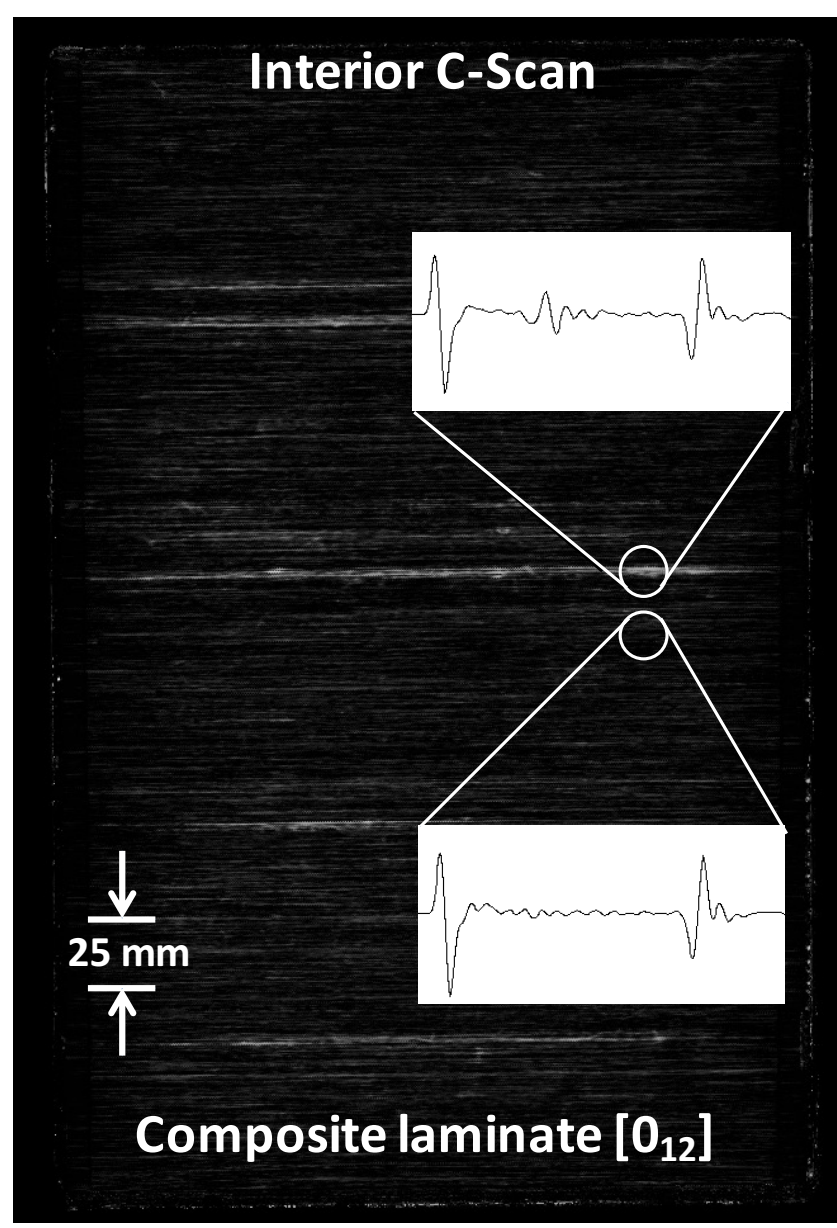

Fig. 2. C-scan of tow-placed laminate having several tow gaps, seen as brighter against the dark background. Sample A-scans at the points indicated, illustrate that the gaps produce reflected echoes without attenuating the back-surface echo.

of resin. As the tow gap gets wider, as in Fig. 3C, the unsupported courses above the gap are likely to sag into the gap volume, resulting in a much more complex resin-filled volume in the cured composite. A photomicrograph of such a case is presented in Fig. 3D. For simplicity, we chose to initially model the simpler physical situation of the narrow gap depicted in Fig. 3B, as layers of composite and resin, limited in one dimension and infinite in the lateral dimensions, and interrogated by a plane ultrasonic wave. In addition, attenuation was not considered.

\section{ONE-DIMENSIONAL MODEL}

The one-dimensional model of an immersion ultrasonic pulse-echo measurement of a tow gap is illustrated in Fig. 4. The nominal condition is given in Fig. 4A, where all tows are properly abutted and no gaps exist. A unit-amplitude ultrasonic plane wave is incident from the left upon the water/composite interface, where it is partially reflected, with reflection coefficient $R_{w c}$, and partially transmitted, with transmission coefficient $T_{w c}$. The first reflected wave is received at the transducer and comprises the front surface signal $S_{\text {front }}$. The transmitted portion continues through the thickness of the composite, is reflected from the back surface with reflection coefficient $R_{c w}$, returns back though the composite and transmits through the front surface again $\left(T_{c w}\right)$ to the transducer, where it comprises the back-surface signal $S_{\text {back. }}$

Neglecting attenuation and scattering from fibers and interlaminar interfaces, for a unit-amplitude incident wave, the front- and back-surface signals are given by

$$
S_{\text {front }}=R_{w c}, \quad S_{\text {back }}=T_{w c} R_{c w} T_{c w}
$$

Fig. 4B depicts the composite with a thin layer of resin, of thickness $d$, representing the tow gap. We treated the thin layer as a single reflector, using the theory developed by Brekhovskikh [4]. The echo from the layer $\left(S_{\text {layer }}\right)$, is built up from transmission into the composite through the front surface $\left(T_{w c}\right)$, reflection from the layer $\left(R_{\text {layer }}\right)$, and transmission out of the composite into the water $\left(T_{c w}\right)$. The back-surface echo $\left(S_{\text {back, }}\right.$, where the prime denotes the presence of the resin layer, to distinguish it from the case in Fig. 4A) now must pass twice through the layer of resin, and so accumulates two factors of the transmission coefficient through the layer $\left(T_{\text {layer }}\right)$, which is symmetric with respect to propagation direction. The results are

$$
\begin{gathered}
S_{\text {front }}=R_{w c}, \quad S_{\text {layer }}=T_{w c} R_{\text {layer }} T_{c w}, \\
S_{\text {back }}^{\prime}=T_{w c} T_{\text {layer }} R_{c w} T_{\text {layer }} T_{c w} .
\end{gathered}
$$

The various reflection and transmission coefficients are determined by the characteristic impedances $\left(Z_{x}\right)$ of the materials, which are the products of the density $\left(\rho_{x}\right)$ times the longitudinal wave speed $\left(v_{x}\right)$ of the material, with the

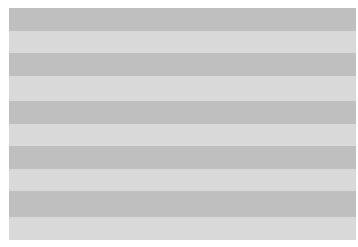

C) Misplacement Larger Gap Width

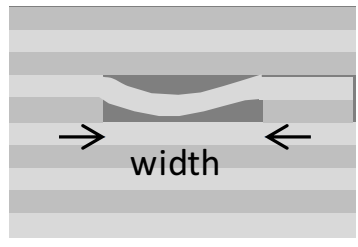

\section{A) Nominal Placement Zero Gap Width}

B) Misplacement Small Gap Width

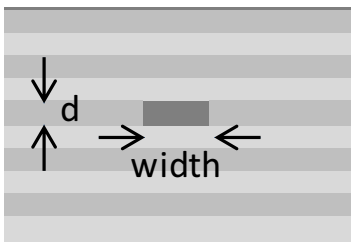

D) Photomicrograph Larger Gap Width

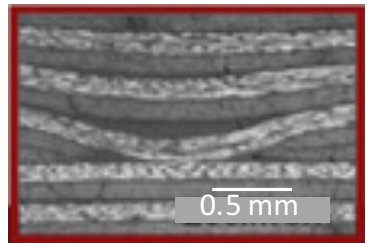

Fig. 3. Schematic drawings of cross-sections of a tow gap. A) Nominal placement with the tows abutted together with zero gap. B) A relatively small gap, with gap width of approximately three ply thicknesses. C) A larger gap, showing the sagging of overlying fiber plies into the gap, forming a resin volume of complex shape. D) A photomicrograph of an example of the case C). (Used by permission [2]). 

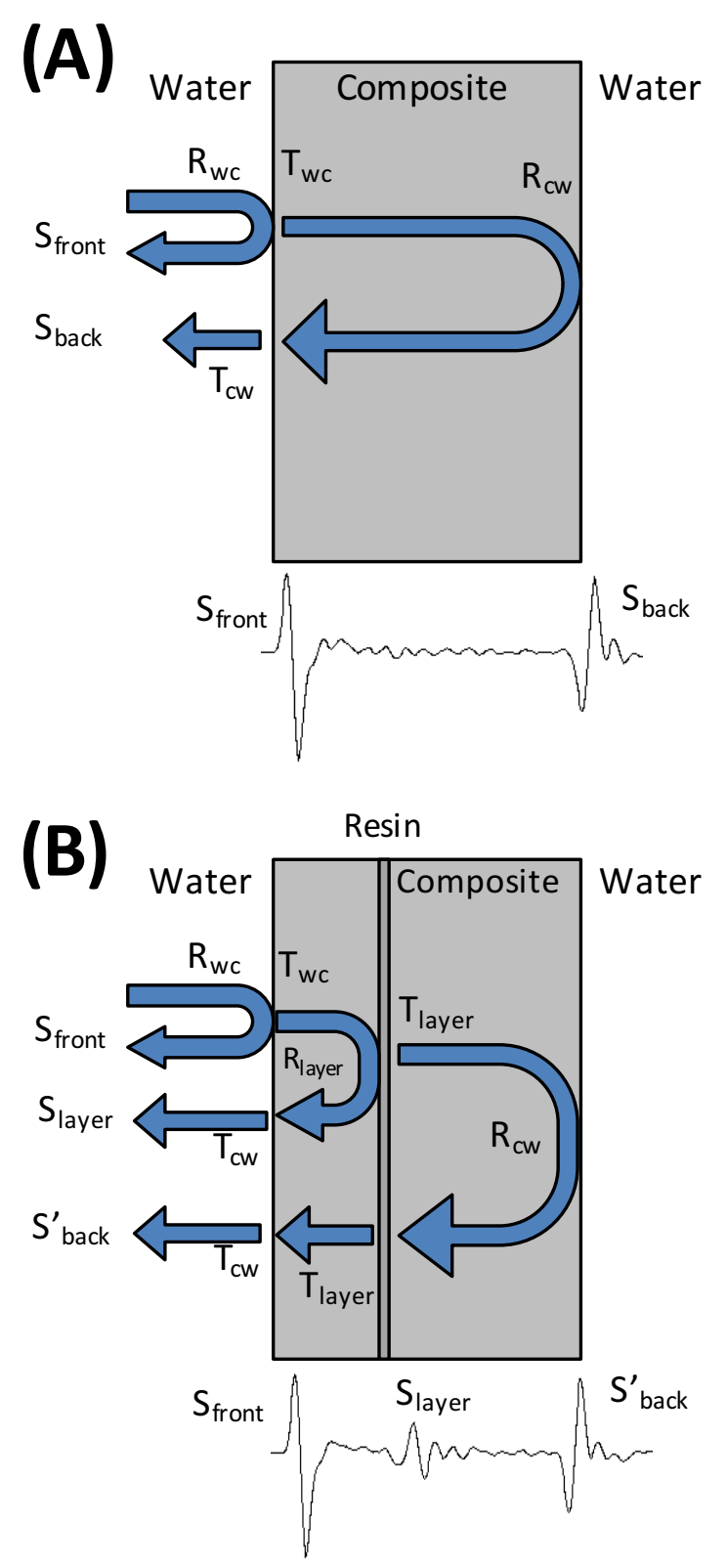

Fig. 4. Schematic of the one-dimensional model. (A) Composite in water, showing reflections and transmissions at front surface and back surface. (B) Composite with thin layer of resin in water. Reflections and transmissions are shown at front surface, back surface, and the thin layer as an entity.

subscript taking the values $(w, c, r)$ to correspond to water, composite, and resin, respectively. Referring to the theory of reflection from a layer [4], the coefficients for a layer of thickness $d$ and measured at ultrasonic frequency $f$ are:

$$
R_{w c}=\frac{z_{c}-z_{w}}{z_{c}+Z_{w}}, \quad T_{w c}=\frac{2 z_{c}}{z_{c}+Z_{w}}
$$

$$
\begin{gathered}
R_{c w}=\frac{Z_{w}-Z_{c}}{Z_{c}+Z_{w}}, \quad T_{c w}=\frac{2 Z_{w}}{Z_{c}+Z_{w}}, \\
R_{r c}=\frac{Z_{c}-Z_{r}}{Z_{c}+Z_{r}}, \quad R_{c r}=\frac{Z_{r}-Z_{c}}{Z_{c}+Z_{r}} \\
R_{\text {layer }}=\frac{z_{c}^{2}-Z_{r}^{2}}{Z_{c}{ }^{2}+Z_{r}^{2}+2 i Z_{c} Z_{r} \cot \left(2 \pi f d / v_{r}\right)}, \\
T_{\text {layer }}=\frac{4 Z_{c} Z_{r}}{\left(Z_{c}+Z_{r}\right)^{2}} \frac{1}{e^{-i 2 \pi f d / v_{r}+R_{c r} R_{r c} e^{i 2 \pi f d / v_{r}}} .}
\end{gathered}
$$

The reflection from and transmission through the resin layer contains modulation terms which provide the interesting physics of this measurement situation.

\section{RESULTS}

\section{A. Experimental Results}

A C-scan of a composite laminate (12 unidirectional plies Hexcel IM7/8552-2) having suspected tow gaps is presented in Fig. 5A. The data was acquired in immersion with a 10$\mathrm{MHz}, 0.5$-inch diameter, 2-inch focus transducer. The data imaged is the root-mean-square value of the RF signal near the mid-plane of the laminate, indicated by a shaded area, labeled $S_{\text {layer }}$, in the overlying example waveform. The signal level is expressed as $\mathrm{dB}$ relative to the RMS of the front surface echo. A tow gap area is indicated by a rectangle labeled GAP and an area where no gap is suspected is indicated by a rectangle labeled NO GAP. Data from the center of the GAP and the NO GAP rectangles are plotted in Fig. 5B and Fig. 5C, respectively. In each graph, the upper trace is taken from a time gate, centered on the back-surface echo, $S_{\text {back }}$ or $S_{\text {back }}$. The lower trace is taken from the interior $S_{\text {layer }}$ time gate. Note that for the NO GAP area, this data is a measure of the background scattering level $S_{\text {scat }}$.

The data show that the back-surface echo is not significantly changed by the presence of a gap; $-2.6 \mathrm{~dB}$ is practically identical to $-3.0 \mathrm{~dB}$. Also, the data show that echoes arising from the gap are, on average, about $6 \mathrm{~dB}$ higher than the surrounding scattering from fibers, and the gap echoes are highly variable, ranging from a "down in the noise" $-25 \mathrm{~dB}$ to a substantial $-12 \mathrm{~dB}$.

\section{B. Model Results}

The model calculation required values for the various material properties involved. For water, we used the usual values $\rho_{w}=1.0 \mathrm{~g} / \mathrm{cm}^{3}$ and $v_{w}=0.148 \mathrm{~cm} / \mu \mathrm{sec}$. From the Hexcel product data sheets [5], we derived $\rho_{r}=1.3 \mathrm{~g} / \mathrm{cm}^{3}$ and $v_{r}=0.189 \mathrm{~cm} / \mu \mathrm{sec}$ for the neat resin. Experimental acid resin digestion measurements of portions of this specimen yielded $\rho_{c}=1.6 \mathrm{~g} / \mathrm{cm}^{3}$ and $61 \%$ volume fraction, given a density of $1.78 \mathrm{~g} / \mathrm{cm}^{3}$ for IM7 fibers, and our measurements gave $v_{c}=$ $0.23 \mathrm{~cm} / \mu \mathrm{sec}$ for the composite. A nominal ply thickness of $0.0127 \mathrm{~cm}$ was used. 
The echo signals $S_{\text {front }}, S_{\text {back }}, S_{\text {back }}(d, f)$, and $S_{\text {layer }}(d, f)$ were calculated using (1-8) over 200 frequencies spanning $8.0-12.0 \mathrm{MHz}$, representing the bandwidth of the measuring transducer, and for 200 layer thicknesses ranging from $0-$ $0.0150 \mathrm{~cm}$. The values of $S_{\text {back }}$, and $S_{\text {layer }}$ were averaged over frequency, for comparison with the measured values which were effectively integrated over frequency. The results are plotted in Fig. 6.
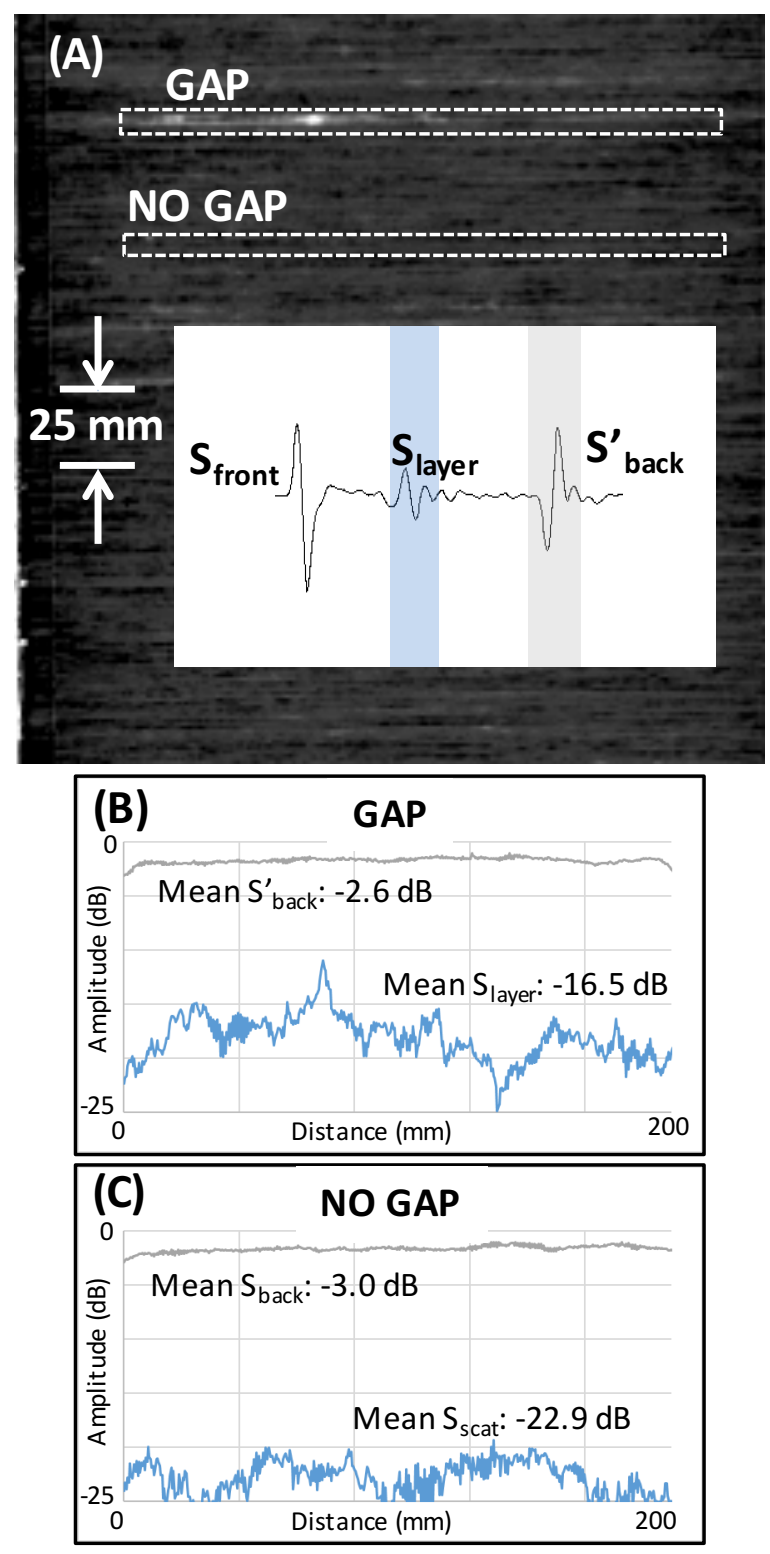

Fig. 5. Experimental data: (A) A segment of C-scan indicating an area having an apparent tow gap and an area with no apparent tow gap. Inset is an A-scan indicating the time gates used to measure the RMS values of the gap echo $\left(\mathrm{S}_{\text {layer }}\right)$ and the back-surface echo $\left(\mathrm{S}_{\text {back }}\right.$ or $\left.\mathrm{S}_{\text {back }}\right)$. (B) Data from on the tow gap. (C) Data not on the tow gap. Note that here, the $\mathrm{S}_{\text {layer }}$ time gate is measuring the background scattering.

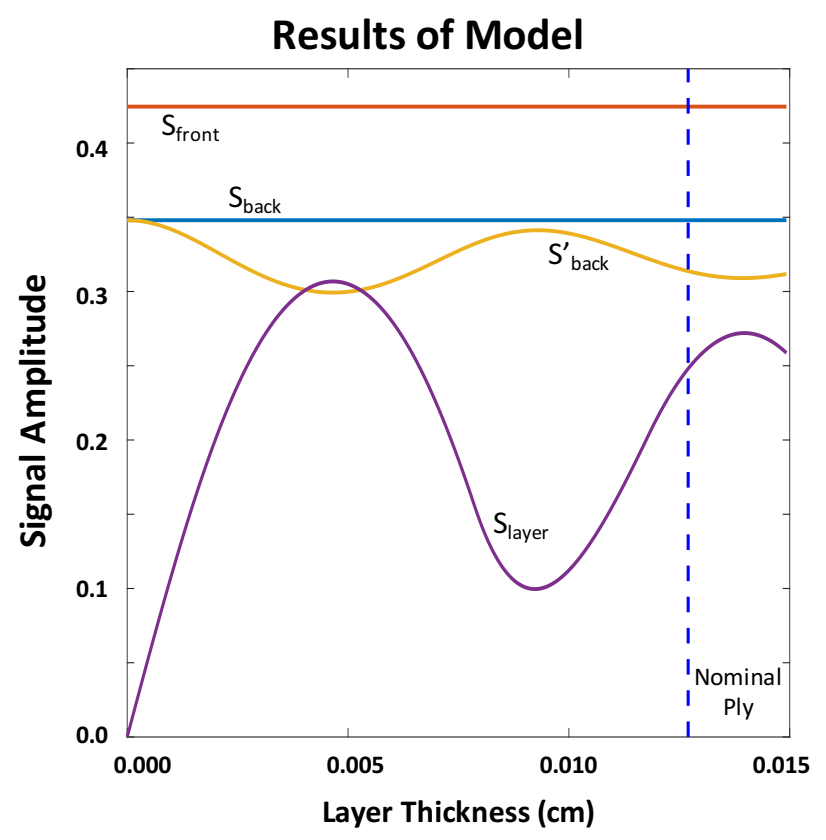

Fig. 6. Results of model for resin layer thickness ranging from zero to $0.015 \mathrm{~cm}$, which is slightly larger than one nominal laminae thickness.

\section{Model Results}

Fig. 6 presents the results for $S_{\text {front }}, S_{\text {back }}, S_{\text {back, }}$, and $S_{\text {layer }}$ calculated from the model as solid lines, as a function of resin layer thickness. The front surface echo $S_{\text {front }}$ and the backsurface echo in the absence of a resin layer $S_{\text {back }}$ are constant at 0.42 and 0.35 , respectively. The echo from the resin layer $S_{\text {layer }}$ and the back-surface echo with a resin layer $S_{\text {back }}$ each exhibit modulation with layer thickness.

\section{Comparison of Results}

The results of model and experiment are compared in Fig. 7 and Fig. 8. In these figures, the logarithmic experimental values given in Fig. 5 have been linearized relative to the front surface reflection from the model and superimposed over the model results as bands with dashed lines, representing the mean and the maximum and minimum of each quantity.

1) Magnitude of Measured and Modeled Back Surface Echoes: The experimental back surface echoes are practically identical, and fall just below the value 0.35 obtained by the model for the layer-free case. This difference is likely due to the attenuation in the composite, which was not included in the model, and effects due to the finite width of the tow gap and the beam width, which are not manifest in the plane wave model.

2) Relative Magnitude of $S_{b a c k}$ and $S_{\text {back }}$ : The measured back-surface echoes are very similar in magnitude, suggesting that the tow gap exhibited a nearly unity transmission coefficient. However, the modeled echo which passes through 


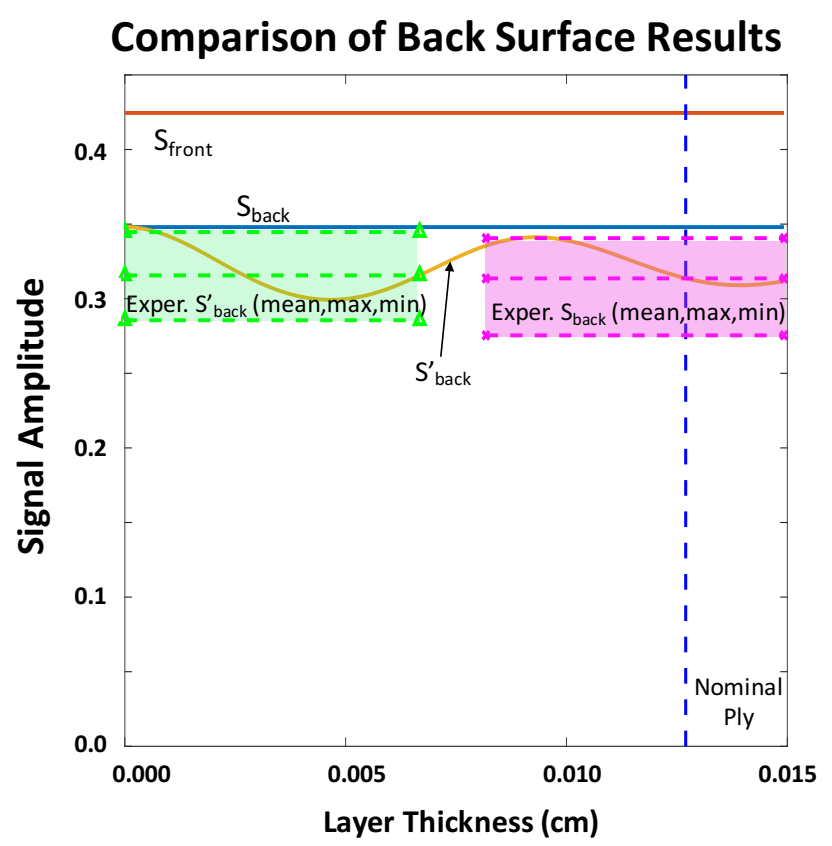

Fig. 7. Comparison of model results and experimental results for the back-surface echo.

the resin layer oscillates dramatically with changing layer thickness, dipping to as low as about $85 \%$ of the echo without a layer. The cause of the difference is likely the finite size of the physical tow gap and the measuring ultrasonic beam. Considering that the measured $6 \mathrm{~dB}$ beam width for the transducer used was $0.061 \mathrm{~cm}$, and that actual tow gaps are likely only several ply thicknesses $(0.0127 \mathrm{~cm})$ wide, the measured back surface echo over a gap is a superposition of waves passing through the gap and waves passing around the gap undisturbed. The model assumes a plane wave and infinite lateral extent of the layer, which is not representative of the measurement.

3) Reflection from the layer: The model reflection from the layer varies widely over the layer thickness range shown in Fig. 8, rising to nearly 0.3 then dipping close to 0.1 before rising rapidly again, with a mean value of approximately 0.2 . The measured reflection from a tow gap varies over a slightly smaller range, about $0.03-0.17$, overlapping the values from the model, with a mean value of 0.06 . The difference between means is consistent with the lack of attenuation in the model. It is apparent that any small variations in thickness of the resin layer would result in relatively large changes in the reflected magnitude. In addition, any changes in the gap width, or effective gap width resulting from the sagging of fibers from above, might also cause echo magnitude fluctuations.

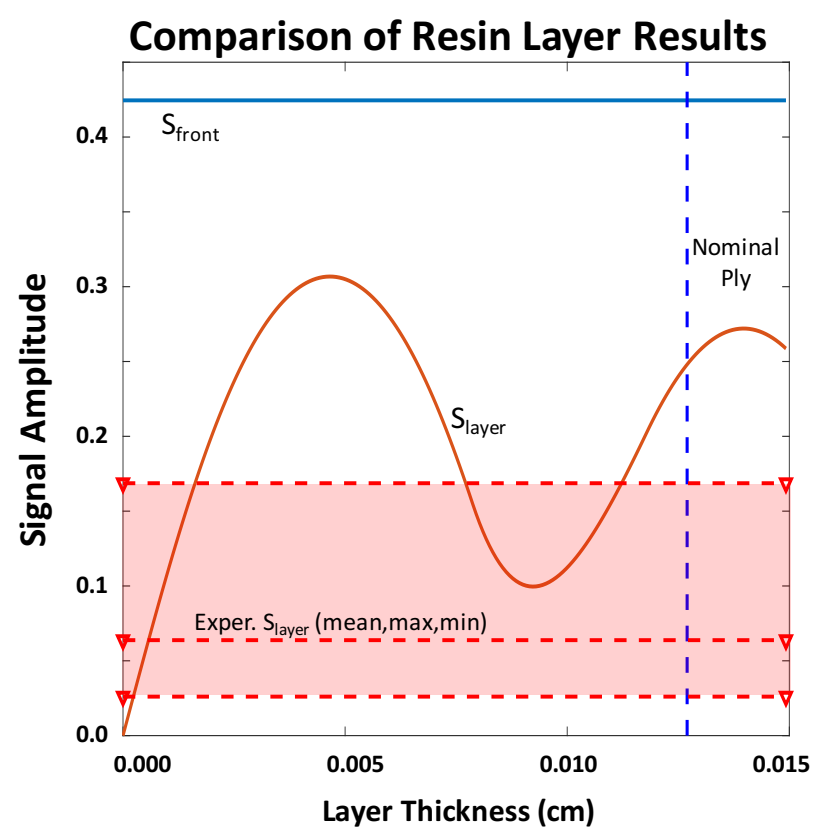

Fig. 8. Comparison of model results and experimental results for the resin layer.

\section{CONCLUSION}

Pulse-echo ultrasonic measurements of tow gaps in towplaced composite laminates were observed to have a substantial echo arising from the resin-rich gap volume, but not have a diminished back surface reflection. A simple onedimensional model was employed to describe the reflections from a composite having a thin resin layer. The results of the model qualitatively match the observed behavior, in which the resin layer causes a measurably large reflection and a relatively small transmission loss. In the near future, the specimen measured will become available for sacrifice in order to micrograph cross-sections of the suspected tow gaps, which may aid in the interpretation of these results. Future work will incorporate a three-dimensional model and include attenuation for a better fit to measurement.

\section{REFERENCES}

[1] A.W. Blom, C. S. Lopes, P. J. Kromwijk, Z. Gurdal, and P. P. Camanho, "A theoretical model to study the influence of tow-drop areas on the stiffness and strength of variable-stiffness laminates," J. Composite Materials, vol. 43, pp. 403-425, 2009.

[2] K. Croft, L. Lessard, D. Pasini, M. Hojjati, J. Chen, and A. Yousefpour, "Experimental study of the effect of automated fiber placement induced defects on performance of composite laminates," Composites: Part A, vol. 42, pp. 484-491, 2011.

[3] P. D. Juarez, K. E. Cramer, and J. P. Seebo, "Advanced in situ inspection of automated fiber placement systems," SPIE 2016 Thermosense Conference, 2016.

[4] L. M. Brekhovskikh, Waves in Layered Media, $2^{\text {nd }}$ ed., trans. by R. T. Beyer, New York: Academic Press, 1980, chapt. 3.

[5] HexPly_8552_us_DataSheet.pdf and IM7_HexTow_DataSheet.pdf, from http://www.hexcel.com/. 